\title{
Prognostic values of serum IP-10 and IL-17 in patients with pulmonary tuberculosis
}

\author{
Yung-Che Chen ${ }^{\mathrm{a}, \mathrm{b}, \mathrm{c}, 1}$, , Chien-Hung Chin ${ }^{\mathrm{a}, 1}$, Shih-Feng Liu ${ }^{\mathrm{a}}$, Chao-Chien $\mathrm{Wu}^{\mathrm{a}}$, Chia-Cheng Tsen ${ }^{\mathrm{a}}$, \\ Yi-Hsi Wang ${ }^{\mathrm{a}}$, Tung-Ying Chao ${ }^{\mathrm{a}}$, Chien-Hao Lie ${ }^{\mathrm{a}}$, Chung-Jen Chen ${ }^{\mathrm{d}}$, Chin-Chou Wang ${ }^{\mathrm{a}}$ and \\ Meng-Chih Lin ${ }^{\mathrm{a}, \mathrm{e}, *}$ \\ ${ }^{a}$ Division of Pulmonary and Critical Care Medicine, Department of Internal Medicine, Chang Gung Memorial \\ Hospital-Kaohsiung Medical Center, Chang Gung University College of Medicine, Kaohsiung, Taiwan \\ ${ }^{\mathrm{b}}$ Graduate Institute of Clinical Medical Sciences, Chang Gung University College of Medicine, Kaohsiung, Taiwan \\ ${ }^{\mathrm{c}}$ Chang Gung Technology Institute, Gia-Yi, Taiwan \\ ${ }^{\mathrm{d}}$ Division of Rheumatology, Chang Gung Memorial Hospital-Kaohsiung Medical Center, Chang Gung University \\ College of Medicine, Kaohsiung, Taiwan \\ eXiamen Chang Gung Hospital, Xiamen, China
}

\begin{abstract}
Objective: To identify patients at high risk of relapse after anti-tuberculosis (TB) therapy or with poor long-term outcomes.

Methods: Fifty-one patients with pulmonary TB: 7 were classified as high association with both cavitations on initial chest radiography and positive sputum smear/cultures after two months of anti-TB treatment (HA group); 19 medium association (MA, one risk alone); and 25 low association (LA, neither risk). Serum interferon (IFN)- $\gamma$-inducible protein 10 (IP-10), interleukin-17 (IL-17), and C-reactive protein levels were investigated.

Results: There was a trend towards higher serum IP-10 levels $(p=0.042)$ for HA patients throughout the 6-month treatment period. Month-2 IP-10 levels were higher in the HA than in the MA/LA group (656.2 \pm 234.4 vs. $307.6 \pm 258.5 \mathrm{pg} / \mathrm{ml}$, adjusted $p=0.005$ ). Receiver operating characteristic curves showed that the risk of relapse was well-captured by month-2 IP-10 levels at a cut-off value of $431 \mathrm{pg} / \mathrm{ml}(\mathrm{AUC}=0.857,95 \% \mathrm{CI} 0.75-0.97, p=0.003)$. Month-2 serum IL-17 levels were lower in non-survivors than survivors $(15.7 \pm 2.9 \mathrm{pg} / \mathrm{ml}$ vs. $24.6 \pm 8.2 \mathrm{pg} / \mathrm{ml}, p=0.001)$. Multivariate analysis demonstrated that a month-2 serum IL-17 level of $\leqslant 17 \mathrm{pg} / \mathrm{ml}(p=0.026)$ was independently associated with all-cause mortality.

Conclusions: Serum IP-10 and IL-17 levels after 2 months of anti-TB treatment may be biomarkers for estimating risk of both cavitation and delayed sputum conversion, and for predicting long-term mortality, respectively.
\end{abstract}

Keywords: Pulmonary tuberculosis, interferon- $\gamma$-inducible protein 10 , interleukin-17, caviation, mortality

\section{Introduction}

Standard anti-tuberculosis (TB) therapy consists of six months of rifampin (RIF) and isoniazid (INH), initially supplemented by two months of pyrazinamide (PZA) and ethambutol (EMB) [1]. The presence of both cavitations and a positive smear/culture for $M y$ cobacterium tuberculosis after two months of treatment

\footnotetext{
${ }^{1}$ Y.C. Chen and C.-H. Chin contributed equally to the article.

* Corresponding author: Dr. Meng-Chih Lin, Division of Pulmonary and Critical Care Medicine, Department of Medicine, Chang Gung Memorial Hospital, 123, Ta-Pei Rd, Niao-Sung Hsiang, Kaohsiung Hsien, Taiwan. Tel.: +8867 7317123 ext. 8300; Fax: +8867 7322402; E-mail: linmengchih@hotmail.com.
}

can amplify the risk of relapse to $20 \%$ and above within two years, compared with approximately $5 \%$ for patients with only one risk factor and less than $2 \%$ for patients with neither risk factor [1-6]. The high relapse rate can be reduced by prolongation of the continuation phase from four to six or seven months [7-10]. The American Thoracic Society has recommended extending 6-month regimens by three months if both risk factors are present [1]. The requirement for biomarkers in assessing treatment response to TB stems from its long and variable natural history, and the non-replicating mycobacterial subpopulations that persist in granulomas and large cavities. Biomarkers that can identify patients at high risk of relapse or long-term outcomes may help in making a clinical judgment on the require- 
ment of prolonged or extensive regimens, and in accelerating drug development by providing validated surrogate endpoints. Some biomarkers have been identified as indicators of bacterial persistence or treatment response, but not yet validated in clinical practice [11, 12].

Interferon (IFN)- $\gamma$-inducible protein 10 (IP-10) is a chemokine expressed in both lymphocytes and monocytes after paracrine stimulation of T-cells by IFNs and other proinflammatory cytokines $[13,14]$. IP-10 is involved in trafficking monocytes and activated Thelper cell type 1 cells to inflamed foci through interaction with the CXC chemokine receptor (CXCR) $3[15,16]$. IP-10 has been identified as an indicator for both infection and curability, but not activity or severity of pulmonary TB in previous studies [17-19]. Interleukin (IL)-17 is produced by a subset of activated CD4+ T cells (Th17), as well as some CD8+ T cells, $\gamma \delta \mathrm{T}$ cells, and NKT cells [8-23]. IL-17 may bind to specific receptors (IL-17R) expressed on leukocytes, epithelial cells, vascular endothelial cells, fibroblasts, and mesothelial cells, resulting in neutrophil homeostasis and recruitment through induction of proinflammatory cytokines and chemokine release [2629]. Up-regulation of IL-17 expression in T lymphocytes has been correlated with active TB [30]. Creactive protein (CRP) is a non-specific marker of systemic inflammation, and has been linked to the presence of cavitations and treatment response to pulmonary TB [31-33]. The roles of these three serum proteins in estimating risk of cavitation and/or a positive sputum smear/culture or predicting long-term outcomes in pulmonary TB have not yet been determined.

In this study, we investigated whether these serum proteins could serve as biomarkers associated with high risk of cavitation and/or a positive sputum smear/culture after 2-month anti-TB treatment, or linked with poor long-term outcomes, such as death. Therefore, we prospectively followed up these parameters in active pulmonary TB patients for a 6-month treatment period, and correlated them with either the presence of risk factors for relapse (cavitations on chest radiographs (CXR) at diagnosis and a positive sputum smear/culture after two months of anti-TB treatment), or all-cause mortality.

\section{Patients and methods}

One hundred and twenty-six patients with active pulmonary TB were enrolled prospectively from our out- patient Pulmonary Clinic at Kaohsiung Chang Gung Memorial Hospital, a medical centre in southern Taiwan, from July 2006 to December 2008. The diagnostic criteria were based on the guidelines of the American Thoracic Society (ATS) [1]. Patients who had true non-tuberculous mycobacterium (NTM) infection (12 patients), poor drug compliance (15 patients), immunocompromised status as a result of human immunodeficiency virus infection ( 2 patients), and those receiving immunosuppressive agents (4 patients), were excluded. Figure 1 shows the flow chart of how the patients were included and classified into groups of high association (HA), medium association (MA), and low association (LA) with risks of relapse. The groups were defined as follows: HA group by the presence of both initial cavity and positive sputum smear/culture after two months of anti-TB treatment; MA group by the presence of one risk factor alone; and LA group by neither risk factor. A total of 51 patients completed follow-up and were included for final analysis. Pulmonary TB was diagnosed by CXR and clinical manifestations, plus a positive sputum culture. Chest computerized tomography was performed for the patients in the HA group to confirm the presence of cavitations in the lung parenchyma. The study protocol was approved by the Institutional Review Board of Kaohsiung Chang Gung Memorial Hospital, and informed consent was obtained from all patients.

\subsection{Processing of sputum samples for AFB smears and mycobacterial cultures}

Concentrated sputum smears were examined for acid fast staining bacilli (AFB) at diagnosis and after 2month anti-TB treatment for each patient, using both the Ziehl-Nielsen and auramine-rhodamine stains. The results were reported using the grading scale of the International Union against Tuberculosis and Lung Disease. A laboratory certified for mycobacteriological diagnostics performed homogenization, decontamination, and cytocentrifugation before culture. The culture techniques used both solid (LJ) and liquid (MGIT) media following standard procedures [34,35].

\section{2. $C X R$ grading of disease}

Standard posterio-anterior CXR were taken at diagnosis and after two and six months of anti-TB treatment in all enrolled patients. Text book grading for pulmonary involvement was adopted for disease assessment [36]. A minimal lesion was defined as slight-to- 


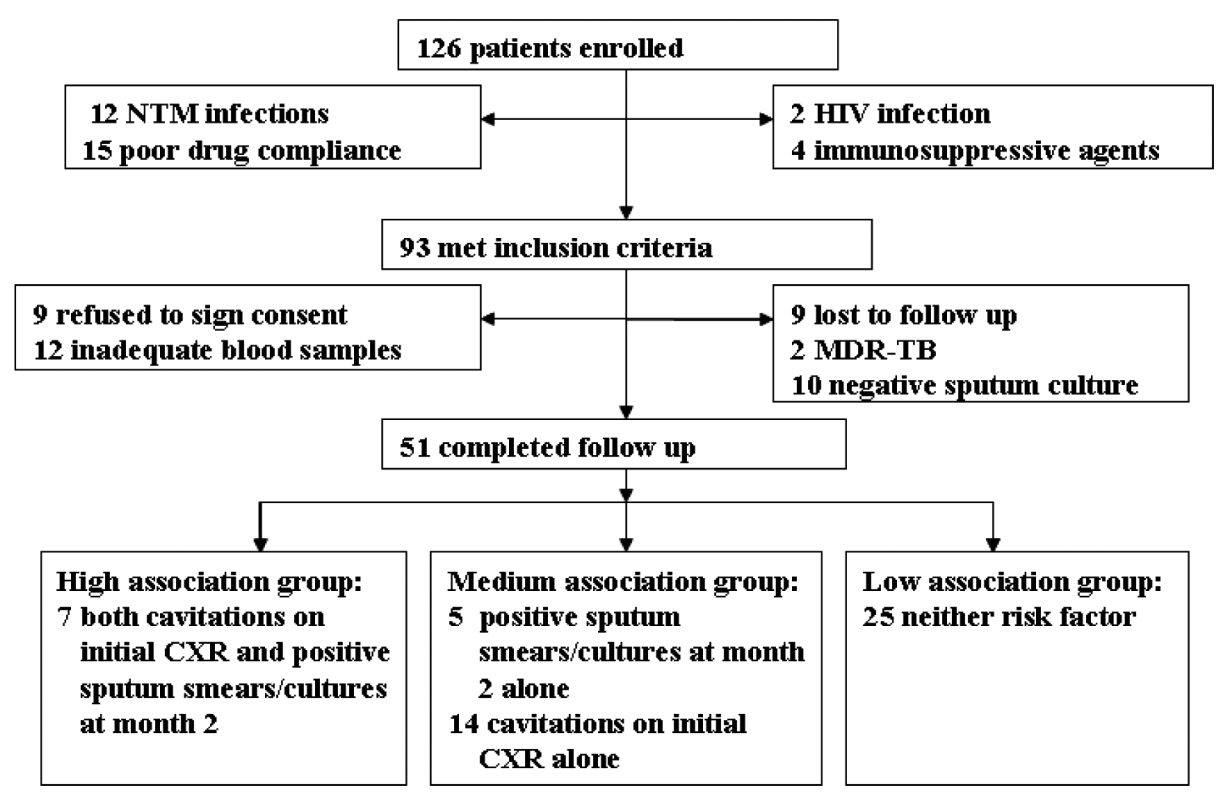

Fig. 1. Flow chart to show classification profiles. M.tb = Mycobacterium tuberculosis; NTM = non-tuberculous mycobacterium; HIV = human immunocompromised virus; $\mathrm{MDR}=$ multidrug resistant.

moderate density not containing demonstrable cavitation with total extent not exceeding the lung volume on one side above the chondro-sternal junction. A faradvanced lesion was defined as slight-to-moderate density that extended more than the total volume of one lung or an equivalent in both lungs; the total diameter of the cavitation, if present, had to be more than $4 \mathrm{~cm}$. Delayed resolution on CXR at month 2 was defined as a reduction of all the lesions by less than half after two months of treatment.

\subsection{Treatment}

All patients were treated in accordance with the American Thoracic Society guidelines for the management of TB [1]. Briefly, first line drugs were given in the following daily doses in combinations: Isoniazid (300 mg), Rifampin (10 mg/kg), Pyrazinamide (1$1.5 \mathrm{~g})$, and Ethambutol (15-20 mg/kg). Second line drugs, including streptomycin and fluoroquinolones were substituted for one or more of the first line drugs in special situations such as drug intolerance or resistance. Every patient received direct observed treatment, short-course (DOTS) strategy and regular followup at our Pulmonary Clinic. Patients were managed for at least six months or longer depending on the presence of cavitations or extent on CXR, 2-month sputum smear/culture results, and clinical response to treatment.

\subsection{Measurements of $I P-10, I L-17$, and CRP in the serum}

IP-10 and IL-17 levels were assessed by a commercial ELISA method (R\&D systems, Minneapolis, $\mathrm{MN})$. All samples were assayed in duplicate according to the manufacturer's protocol. Hypersensitivity CRP (hsCRP) levels were measured by nephelometry (Dade Behring BM2, Marburg, Germany) according to standard procedures with a functional sensitivity of 0.18 $\mathrm{mg} / \mathrm{L}$. Blood samples at diagnosis within two weeks of anti-TB treatment (month 0) and after two months of anti-TB treatment (month 2) were obtained and analyzed from all patients (7 HA, 19 MA, 25 LA), and after six months of anti-TB treatment (month 6) from 35 patients (6 HA, $12 \mathrm{MA}, 15 \mathrm{LA}$ ).

\subsection{Statistical analysis}

Continuous values were expressed as mean \pm standard deviation (SD). The differences between the HR, MR, and LR groups were analyzed by Mann-whitney, Wilcoxon ranked sum, Kruskal-Wallis $\mathrm{H}$, and chisquare tests, since they were not normally distributed. To allow random effects to be properly specified and to handle some missing data in the 6-month longitudinal experiments, mixed model analysis was used to compare blood levels between the HA and MA/LA groups at three different time points. To investigate predic- 
Table 1

Comparison of the characteristics between high association (HA) and medium/low association (MA/LA) groups

\begin{tabular}{|c|c|c|c|}
\hline Characteristics & HA group $N=7$ & MA/LA group $N=44$ & $P$ value \\
\hline Age, years & $52.1 \pm 15.0$ & $57 \pm 18.6$ & 0.434 \\
\hline Male, n (\%) & $6(85.7)$ & $32(72.7)$ & 0.516 \\
\hline \multicolumn{4}{|l|}{ Co-morbidity, n (\%) } \\
\hline Diabetes mellitus & $5(71.4)$ & $12(21.3)$ & 0.021 \\
\hline COPD & $2(28.6)$ & $4(8.9)$ & 0.129 \\
\hline Chronic renal failure & $0(0)$ & $5(11.1)$ & 0.354 \\
\hline Chronic hepatitis & $0(0)$ & $1(2.2)$ & 0.69 \\
\hline Congestive heart failure & $0(0)$ & $2(4.4)$ & 0.569 \\
\hline Malignancy & $0(0)$ & $6(13.3)$ & 0.304 \\
\hline Smoking history, n (\%) & $6(85.7)$ & $22(50)$ & 0.078 \\
\hline Alcoholism, n (\%) & $2(28.6)$ & $10(22.7)$ & 0.735 \\
\hline Systemic symptoms, n (\%) & $6(85.7)$ & $27(60)$ & 0.189 \\
\hline \multicolumn{4}{|l|}{ Sputum at diagnosis, n (\%) } \\
\hline Smear AFB positive & $6(85.7)$ & $32(72.8)$ & 0.446 \\
\hline $1+$ & $1(14.3)$ & $8(18.2)$ & \\
\hline $2+$ & $0(0)$ & $4(9.1)$ & \\
\hline $3+$ & $5(71.4)$ & $20(45.5)$ & \\
\hline Drug-resistant strain & $2(28.6)$ & $8(17.8)$ & 0.5 \\
\hline \multicolumn{4}{|l|}{ CXR at diagnosis, $\mathrm{n}(\%)$} \\
\hline Severity & & & 0.577 \\
\hline Minimal lesions & $0(0)$ & $4(9.1)$ & \\
\hline Moderately advanced & $1(14.3)$ & $10(22.7)$ & \\
\hline Far advanced lesions & $6(85.7)$ & $30(68.2)$ & 0.344 \\
\hline Bilateral involvement & $6(85.7)$ & $31(70.5)$ & 0.401 \\
\hline Cavities & $7(100)$ & $14(31.8)$ & 0.001 \\
\hline Pleural effusion & $1(14.3)$ & $8(18.2)$ & 0.802 \\
\hline Time from starting treatment to collecting blood, days & $3 \pm 5.5$ & $2.3 \pm 4.5$ & 0.825 \\
\hline
\end{tabular}

tive accuracy, the candidate predictor was analyzed by area under the receiver operating characteristic (ROC) curves. Multivariate Cox proportional hazards regression analysis with stepwise forward selection was used to evaluate independent prognostic factors associated with survival, and age, smoking history, and month2 serum IL-17 level with a cut-off value of $17 \mathrm{pg} / \mathrm{ml}$ were used as covariates. The Kaplan-Meier method was used to estimate overall survival. Differences in survival among groups were analyzed with the log-rank test. The null hypothesis was rejected at $p<0.05$. Analyses were performed using the SPSS 15.0 statistical software package (SPSS Corp., Chicago, IL).

\section{Results}

\subsection{Comparisons of clinical characteristics of patients between the HA and MA/LA groups}

None of the serum protein levels measured in the 19 MA patients at any of the 3 time points differed from that of the $25 \mathrm{LA}$ patients, so we lumped them together (MA/LA group). Baseline clinical characteristics of the 51 culture-positive TB patients at high and medium/low risk of relapse are presented in Table 1. A larger proportion of patients in the HA group had diabetes mellitus than that in the MA/LA group ( $p=$ 0.021 ). Treatment responses based on microbiological and CXR findings, durations of individual medication and total treatment course, and all-cause mortality are presented in Table 2. A larger proportion of patients in the HR group had delayed resolution on CXR at month $2(p=0.002)$.

\subsection{Higher serum IP-10 levels at month 2 in the HA group}

There was a trend towards higher serum IP-10 levels ( $p=0.042$, Fig. 2A) for patients in the HA group throughout the 6-month treatment period by the mixed model analysis. In comparisons at individual time points, only month-2 IP-10 levels were significantly higher in the HA group than those in the MA/LA group after adjusting for diabetes mellitus $(656.2 \pm 234.4 \mathrm{vs}$. $307.6 \pm 258.5 \mathrm{pg} / \mathrm{ml}$, adjusted $\mathrm{p}$ value $=0.005)$. In contrast, serum IL-17 and hsCRP levels showed no difference between the HA and MA/LA groups (Fig. 2B and 2C). There was a trend towards lower serum hsCRP levels with time in all the patients during the 6-month 
Table 2

Comparisons of treatment response, medication and outcomes between high association (HA) and medium to low association (MA/LA) groups with pulmonary tuberculosis

\begin{tabular}{lccc}
\hline & HA group $N=7$ & MA/LA group $N=44$ & P value \\
\hline Sputum at month 2, $(\%)$ & & & \\
$\quad$ Smear positive & $5(71.4)$ & $4(10.3)$ & $<0.001$ \\
$\quad$ Culture positive & $2(28.6)$ & $2(4.5)$ & 0.045 \\
$\quad$ Smear or culture positive & $7(100)$ & $5(11.4)$ & $<0.001$ \\
Follow-up CXR findings, n (\%) & & \\
$\quad$ Delayed resolution at month 2 & $6(85.7)$ & $11(25)$ & 0.002 \\
$\quad$ Extensive lesions at month 6 & $3(42.9)$ & $15(35.7)$ & 0.717 \\
Duration of medication, months & & & \\
Isoniazid & $8.1 \pm 1.5$ & $7.1 \pm 2.6$ & 0.291 \\
$\quad$ Rifampin & $8.1 \pm 1.5$ & $7.0 \pm 2.5$ & 0.253 \\
$\quad$ Ethambutol & $6.1 \pm 2.9$ & $6.1 \pm 3.3$ & 0.968 \\
$\quad$ Pyrazinamide & $2.7 \pm 1$ & $3.4 \pm 2.6$ & 0.497 \\
Use of 2nd line medication*, $\mathrm{n}(\%)$ & $2(28.6)$ & $5(11.4)$ & 0.219 \\
Treatment duration $\geqslant 9$ months, $\mathrm{n}(\%)$ & $5(71.4)$ & $25(56.8)$ & 0.466 \\
All-cause mortality, n $(\%)$ & $1(14.3)$ & $4(7.3)$ & $0.625^{* *}$ \\
\hline
\end{tabular}

$\mathrm{CXR}=$ chest radiograph; ${ }^{*}$ Including Moxifloxacine, Levofloxacine, Ciproxine, and Streptomycin;

** Compared by the log rank test.
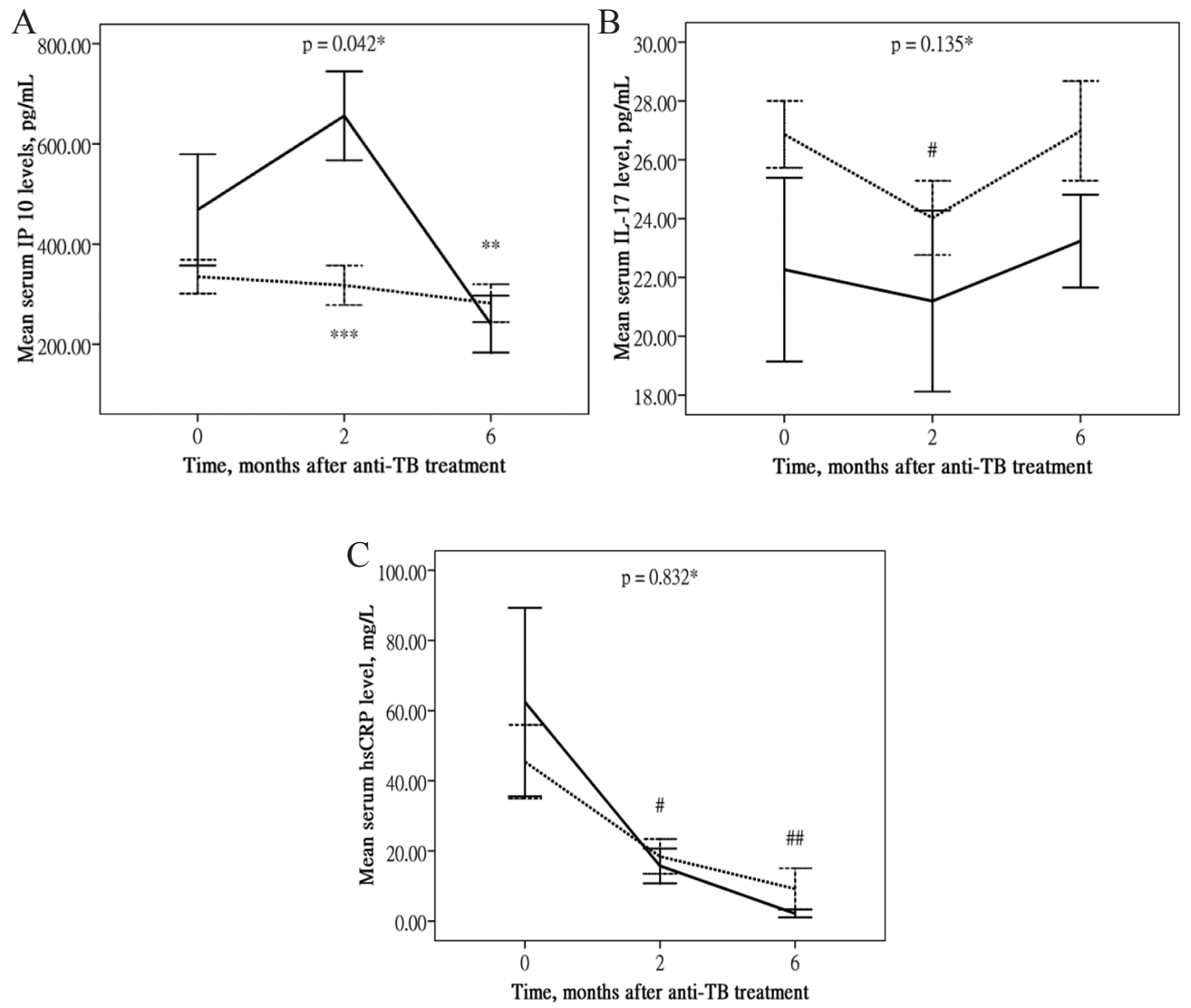

Fig. 2. Relationships between blood levels of IP-10, IL-17, and hsCRP, and risk of caviation and a positive sputum smear/culture after two months of anti-tuberculosis treatment in the study groups. Data are expressed as the mean values of IP-10 (A), IL-17 (B), and hsCRP (C) levels at 0, 2, and 6 months after treatment. Standard errors are indicated by vertical bars. Results of the medium association and low association (MA/LA) groups are shown by the dashed line, and results of the high association (HA) group are shown by the straight line. ${ }^{*}$ Global $\mathrm{p}$ values for the fixed effect of grouping by the mixed model analysis; ${ }^{* *} p<0.05$, month 2 vs. month 6 for the HA group; ${ }^{* * *} p=0.005$, HA vs. MA/LA group at month 2; ${ }^{\#} p<0.005$, month 2 vs. month 0 for the MA/LA group; \#\# $p<0.005$, month 6 vs. month 0 for the MA/LA group. 


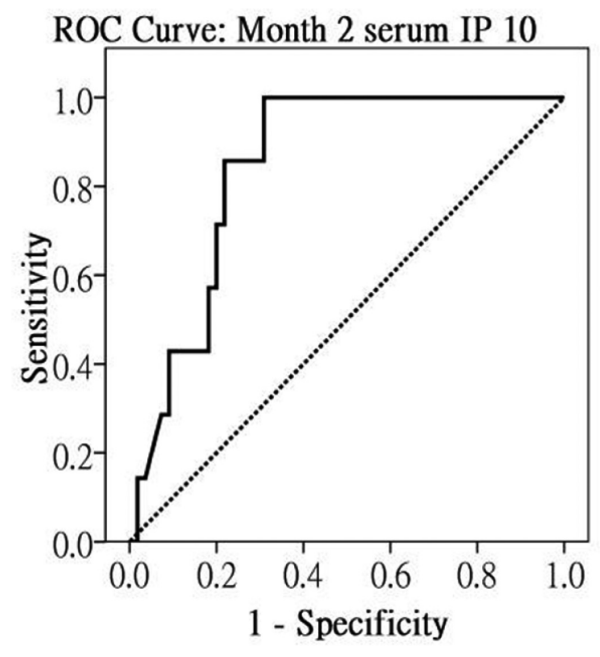

Fig. 3. Predictive accuracy of the serum IP-10 levels measured at month 2. The corresponding ROC curve showed that the risk of relapse was well captured by month-2 IP-10 levels at a cut-off value of $431 \mathrm{pg} / \mathrm{ml}(\mathrm{AUC}=0.857,95 \%$ CI 0.75-0.97, $p=0.003)$.

treatment period by the mixed model analysis ( $p=$ 0.001, Fig. 2C). In comparisons between two contiguous time points, serum hsCRP levels at month 6 were lower than that at month $0(p=0.002)$, and serum IL-17 levels at month 2 were lower than that at month $0(p=0.009)$ only in the MA/LA group. After six months of anti-TB treatment, patients in the HA group had a reduction in their serum levels of IP-10 (661.9 \pm 256.2 vs. $240.4 \pm 138.8 \mathrm{pg} / \mathrm{ml}, p=0.028)$ and hsCRP $(14.19 \pm 12.95$ vs. $2.14 \pm 2.42 \mathrm{mg} / \mathrm{L}, p=0.043)$ compared to those at month 2 . The corresponding ROC curves showed that the risk of both cavitation and a positive sputum smear/culture at month 2 was wellcaptured by month-2 IP-10 levels at a cut-off value of $431 \mathrm{pg} / \mathrm{ml}$ (AUC $=0.857,95 \%$ CI 0.75-0.97, $p=$ $0.003)$, displaying a sensitivity of $85.7 \%$ and specificity of $79.5 \%$ (Fig. 3 ).

\subsection{Correlations of serum IP-10/hsCRP levels at diagnosis with disease severity}

Serum IP-10 levels at diagnosis were significantly higher $(424.4 \pm 236.6$ vs. $251.3 \pm 208.4 \mathrm{pg} / \mathrm{ml}$, adjusted $\mathrm{p}$ value $=0.007)$ ) in patients with high sputum smear grading (sputum AFB 2+ to $3+$ at diagnosis, $N=30$ ) than those with low sputum smear grading (sputum AFB 0 to $1+$ at diagnosis, $N=21$ ), after adjusting for age, CXR grading, and time from starting treatment to collecting blood by linear regression analysis (Fig. 4A). Serum hsCRP levels at diagnosis were significantly higher (63.2 \pm 72.8 vs. $12.5 \pm 26.4 \mathrm{mg} / \mathrm{L}$, adjusted $\mathrm{p}$ value $=0.024)$ in patients with far-advanced lesions on initial CXR $(N=36)$ than those with minimal and moderately advanced lesions $(N=15)$, after adjusting for a past smoking history of more than 10 pack-years, the presence of cavitations on CXR, and time from starting treatment to collecting blood by linear regression analysis (Fig. 4B).

\subsection{Lower serum IL-17 levels at month 2 in non-survivors}

In the MA/LA group, four patients died of pneumonia with sepsis, two during the course of anti-TB treatment, and two after completing anti-TB treatment. In the HR group, one patient died of an unknown cause after completing anti-TB treatment. Serum IL-17 levels at month 2 were significantly lower in non-survivors than survivors $(15.7 \pm 2.9 \mathrm{pg} / \mathrm{ml}$ vs. $24.6 \pm 8.2 \mathrm{pg} / \mathrm{ml}$, $p=0.001$ ) (Fig. 5A). Figure 5B shows the KaplanMeier survival curves for the high and low month-2 IL-17 groups based on a cut-off level of $\leqslant 17 \mathrm{pg} / \mathrm{ml}$. Patients with month-2 IL-17 levels of $\leqslant 17 \mathrm{pg} / \mathrm{ml}$ had significantly higher all-cause mortality than those with $>17 \mathrm{pg} / \mathrm{ml}(p=0.007)$. Table 3 shows the unadjusted and adjusted Cox proportional hazards analyses. On a bivariate level, having an older age (hazard ratio (HR) $1.18,95 \%$ CI 1.02-1.37), a past history of cigarette smoking $>10$ pack-years (HR 5.81, 95\% CI 1.33$25.38)$, and a lower month-2 IL-17 level $\leqslant 17 \mathrm{pg} / \mathrm{ml}$ (HR 11.25, 95\% CI 1.25-101.06) were significantly related to mortality in patients with TB in our samples. Multivariate analysis demonstrated that old age (HR $1.54,95 \%$ CI $0.96-2.48, p=0.076)$ and a month-2 serum IL-17 level of $\leqq 17 \mathrm{pg} / \mathrm{ml}$ (HR $42.57,95 \%$ CI $1.56-1158.67, p=0.026)$ were independently associated with mortality when adjusting for other covariates (Table 3).

\section{Discussion}

We undertook this study to determine candidate biomarkers that can identify $\mathrm{TB}$ patients at high risk of the presence of both cavitations on initial CXR and sputum smear/culture positivity after two months of anti-TB treatment. We found that serum IP-10 levels measured after two months of anti-TB treatment served as a biomarker to identify TB patients with both factors. Additionally, serum IL-17 levels measured after two months of anti-TB treatment served as a biomarker associated with risk of death. 
A

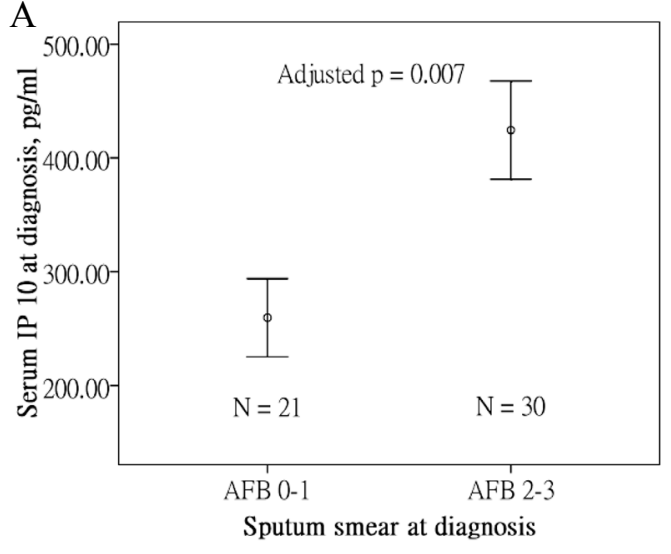

$\mathrm{B}$

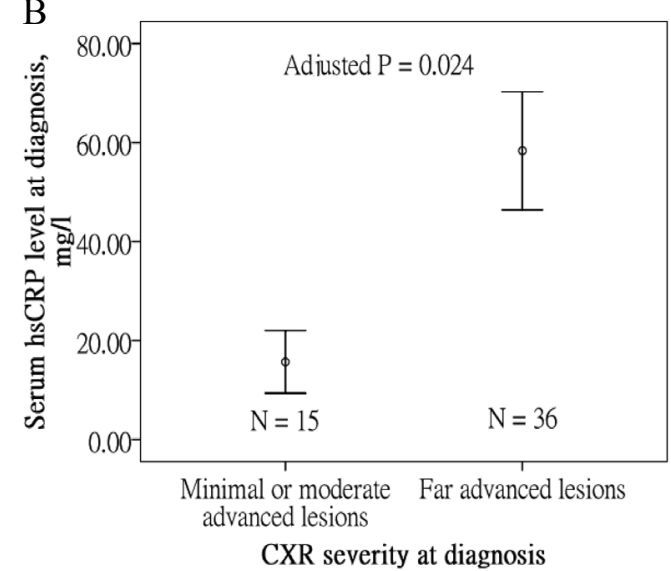

Fig. 4. Relationships between blood levels of IP-10 and hsCRP at diagnosis, and disease activity. (A) Serum IP-10 levels at diagnosis were significantly higher in patients with high sputum smear grading than those with low sputum smear grading. (B) Serum hsCRP levels at diagnosis were significantly higher in patients with far-advanced lesions on initial CXR than those with minimal to moderate-advanced lesions. Data are expressed as the mean values. Standard errors are indicated by vertical bars.

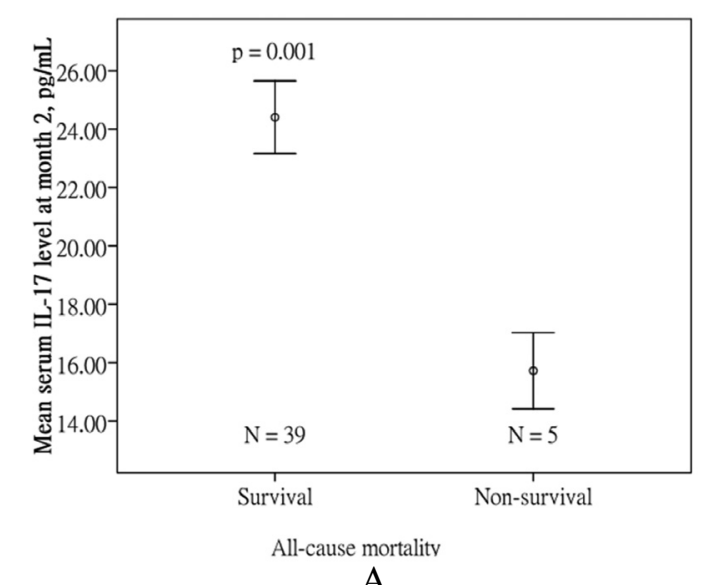

A

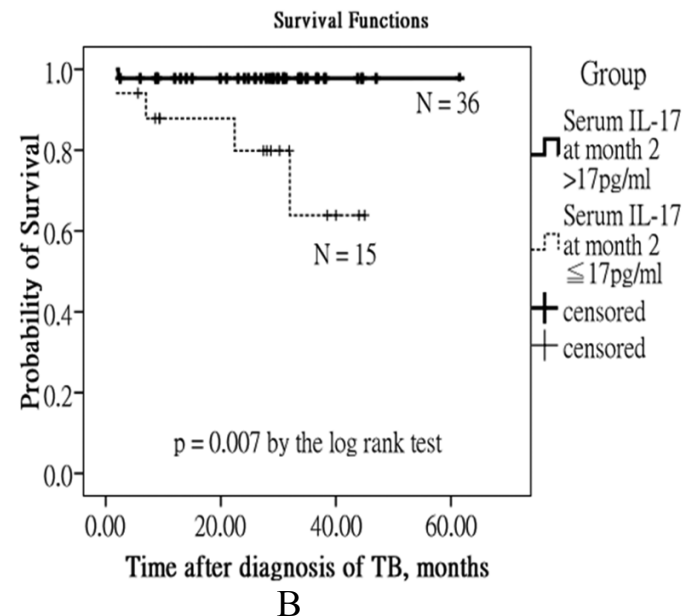

B

Fig. 5. Relationship between blood IL-17 levels measured at month 2 and all-cause mortality in the study population. (A) Serum IL-17 levels at month 2 were significantly lower in non-survivors than survivors. (B) Kaplan-Meier survival curves for the 51 TB patients with separate lines according to serum IL-17 levels at month 2 at a cut-off level of $17 \mathrm{pg} / \mathrm{ml}$.

Serum IP-10 levels were elevated at month 2 in the HR group of patients, but did not differ during the 6month treatment course in the MR/LR group of patients. Initial serum IP-10 levels also correlated with sputum smear grading but not CXR severity at diagnosis. This indicated that IP-10 may represent the burden of Mycobacterium tuberculosis in cavities and lung parenchyma. Accordingly, in a previous study by Azzurri et al., serum IP-10 levels were found to be correlated with the treatment efficacy but not clinical disease severity of pulmonary TB. They observed a significant decline in IP-10 levels after completion of anti-TB treatment in cured patients [17]. In another study by
Whittaker et al., plasma IP-10 levels were found to be even higher in patients with latent TB than those in patients with active TB [18]. IP-10 release from peripheral blood mononuclear cells is strongly induced after $M$. tuberculosis specific stimulation in patients with active and latent TB infections [19]. Taken together, human infection by $M$. tuberculosis may lead to an elevation of IP-10 levels, which can predict disease curability but not activity. In our study, IP-10 levels were not reduced at month 6 in the MA/LA group of patients. This may be contributable to a higher proportion of patients with far-advanced lesions at diagnosis and hence a higher 
Table 3

Cox proportional hazard model to analyze predictors for mortality in all the patients with pulmonary tuberculosis

\begin{tabular}{lcccc}
\hline & Bivariate & & Multivariate \\
& Hazard ratio & $95 \%$ CI & Hazard ratio & 95\% CI \\
\hline Age & 1.18 & $1.02-1.37^{*}$ & 1.54 & $0.96-2.48$ \\
Smoking history & 5.81 & $1.33-25.38^{*}$ & & \\
Sputum AFB at month 0 & 0.9 & $0.47-1.76$ & & \\
CXR grading at month 0 & 36.2 & $0.01-11368.3$ & & \\
Co-morbidity & 3.86 & $0.43-34.69$ & & \\
Cavitation on initial CXR & 0.98 & $0.16-5.85$ & & \\
Positive smear/culture at month 2 & 0.98 & $0.11-8.75$ & & \\
Cavitation and positive smear/culture at month 2 & 1.75 & $0.2-15.73$ & & \\
Month-2 serum IL-17 $17 \mathrm{pg} / \mathrm{mL}$ & 11.25 & $1.25-101.06^{*}$ & 42.57 & \\
\hline
\end{tabular}

$\mathrm{CI}=$ confidence interval; $\mathrm{CXR}=$ chest radiograph. ${ }^{*} p<0.05$.

proportion of patients with extensive lesions at month 6.

None of the patients who finished their anti-TB treatment course developed a clinically detectable relapse, except for one in the HA group who died of an unknown cause. Several factors may explain why relapses did not occur in our study patients. Firstly, $71.4 \%$ of the patients in the HA group and $56.8 \%$ of the patients in the MA/LA group received at least nine months of antiTB treatment. Prolongation of the continuation phase from four to seven months has been demonstrated to be an effective method for reducing the relapse rate in previous studies $[7,8]$. Secondly, patients in both groups received EMB treatment for a mean duration of 6.1 months, rather than just for the initial consolidation phase of two months. Thirdly, all the patients received direct observed treatment as the internationally recommended strategy for TB control [37]. Furthermore, the proportion of patients who received second line medications was greater than $10 \%$, and the proportion of patients who received at least nine months of treatment was greater than $50 \%$ in both the HA and MA/LA groups. This may reflect a higher disease severity in our study population, but good control of the infection under the extended regimens.

Another important finding in this study was that there were lower serum IL-17 levels in non-surviving patients. IL-17 can act to mediate the accumulation of polymorphic and mononuclear cells during mycobacterial infections. IL-17 mRNA levels were more prominently expressed in blood CD8+ T cells from patients with TB when compared to those from healthy subjects [30]. IL-17 levels have been linked to clinical outcomes in several diseases. IL-17-neutralized mice have exhibited significantly greater body weight loss, higher organism growth, and much more severe lung pathology with Chlamydia infection [28]. IL-17 expression has been demonstrated to be an independent prognostic factor for survival in patients with non-small cell lung cancer [38]. Increased allergen-induced IL17 responses during sublingual immunotherapy are associated with elevated symptom scores in allergic children [39]. For the first time, we found that IL-17 may play a role in predicting death in patients with pulmonary TB, although the sample size is relatively small for survival analysis. We speculate that lower serumIL17 levels may reflect a weaker Th1 immune response against mycobacterial infections, since IL-17 is an important cytokine in the induction of optimal Th1 response, the maintainence of the integrity of the granuloma by limiting neutrophil death, and protective immunity against mycobacterial infection [26,27]. Further large scale studies are needed to confirm this association, and the underlying mechanisms by which lower serum IL-17 levels were linked to higher mortality remain to be further investigated.

The present study is biased and has several limitations. The sample size in the HR group was relatively small. However, we estimated the power to be $95.32 \%$ for the comparison of month-2 IP-10 between the HA and MA/LA groups, using the nonparametric test formulations with a simple adjustment to the sample sizes in our study and an $\alpha$ error of 0.05 with PASS 2005 (NCSS, Kaysville, Utah, USA) software. Secondly, there was a large loss to follow up or exclusion in the study population. Some patients were transferred to local physicians or other hospitals because of their preference or convenience. Some patients refused to provide blood samples at the 2 nd or 3 rd time point. Patients with multidrug resistant (MDR)-TB or negative sputum culture were excluded because drug-resistant strains may potentially influence the levels of inflammatory cytokines, and negative culture may raise the uncertainty of clinical diagnosis. Finally, a larger proportion of patients in the HR group had diabetes mellitus. Additionally, some blood samples were not col- 
lected before starting anti-TB treatment, and this would potentially affect the cytokine levels since changes in immune responses might occur as early as 1 week post treatment. However, we have made statistical adjustments for these confounding factors by linear regression analysis.

In conclusion, serum IP-10 levels measured after two months of anti-TB treatment may be a biomarker for estimating the risk of cavitation and a positive sputum smear/culture after 2-month treatment in patients with pulmonary $\mathrm{TB}$, and can be helpful in making an individual-based decision on preventive and therapeutic treatment schemes. In addition, serum IL-17 levels measured after two months of anti-TB treatment may be a biomarker for predicting long-term survival.

\section{Competing interests}

All authors of this paper declare that there is no conflict of interest in this study or in reporting the findings described in this manuscript. No specific funding has been received by any author.

\section{Acknowledgments}

We gratefully acknowledge Professor HL Eng and the clinical staff of the Department of Pathology of the Kaohsiung Chang Gung Memorial Hospital for performing sputum processing. We would also like to kindly acknowledge the patients who agreed to participate in our study.

\section{References}

[1] H.M. Blumberg, W.J. Burman, R.E. Chaisson, C.L. Daley, S.C. Etkind, L.N. Friedman et al., American Thoracic Society/Centers for Disease Control and Prevention/Infectious Diseases Society of America: treatment of tuberculosis, Am J Respir Crit Care Med 167 (2003), 603-662.

[2] M. Zierski, E. Bek, M.W. Long and D.E. Snider, Jr., Shortcourse (6-month) cooperative tuberculosis study in Poland: results 30 months after completion of treatment, Am Rev Respir Dis 124 (1981), 249-251.

[3] D.L. Cohn, B.J. Catlin, K.L. Peterson, F.N. Judson and J.A. Sbarbaro, A 62-dose, 6-month therapy for pulmonary and extrapulmonary tuberculosis. A twice-weekly, directly observed, and cost-effective regimen, Ann Intern Med 112 (1990), 407415.

[4] D.A. Mitchison, Assessment of new sterilizing drugs for treating pulmonary tuberculosis by culture at 2 months, Am Rev Respir Dis 147 (1993), 1062-1063.
[5] D. Benator, M. Bhattacharya, L. Bozeman, W. Burman, A. Cantazaro, R. Chaisson et al., Rifapentine and isoniazid once a week versus rifampicin and isoniazid twice a week for treatment of drug-susceptible pulmonary tuberculosis in HIVnegative patients: a randomised clinical trial, Lancet $\mathbf{3 6 0}$ (2002), 528-534.

[6] R. Singla, D. Srinath, S. Gupta, P. Visalakshi, U.K. Khalid, N. Singla et al., Risk factors for new pulmonary tuberculosis patients failing treatment under the Revised National Tuberculosis Control Programme, India, Int J Tuberc Lung Dis $\mathbf{1 3}$ (2009), 521-526.

[7] K.C. Chang, C.C. Leung, W.W. Yew, S.L. Chan and C.M. Tam, Dosing schedules of 6-month regimens and relapse for pulmonary tuberculosis, Am J Respir Crit Care Med 174 (2006), 1153-1158.

[8] K.C. Chang, C.C. Leung, W.W. Yew, S.C. Ho and C.M. Tam, A nested case-control study on treatment-related risk factors for early relapse of tuberculosis, Am J Respir Crit Care Med 170 (2004), 1124-1130.

[9] Hong Kong Chest service/Tuberculosis Research Centre, Madras/British Medical Research Council, A controlled clinical comparison of 6 and 8 months of antituberculosis chemotherapy in the treatment of patients with silicotuberculosis in Hong Kong, Am Rev Respir Dis 143 (1991), 262-267.

[10] N.N. Bock, T.R. Sterling, C.D. Hamilton, C. Pachucki, Y.C. Wang, D.S. Conwell et al., A prospective, randomized, doubleblind study of the tolerability of rifapentine 600,900 , and $1,200 \mathrm{mg}$ plus isoniazid in the continuation phase of tuberculosis treatment, Am J Respir Crit Care Med 165 (2002), 1526-1530.

[11] F.M. Perrin, M.C. Lipman, T.D. McHugh and S.H. Gillespie, Biomarkers of treatment response in clinical trials of novel antituberculosis agents, Lancet Infect Dis 7 (2007), 481-490.

[12] R.S. Wallis, T.M. Doherty, P. Onyebujoh, M. Vahedi, H. Laang, O. Olesen et al., Biomarkers for tuberculosis disease activity, cure, and relapse, Lancet Infect Dis 9 (2009), 162172.

[13] J.M. Farber, Mig and IP-10, CXC chemokines that target lymphocytes, J Leukoc Biol 61 (1997), 246-257.

[14] V. Gangur, F.E. Simons and K.T. Hayglass, Human IP-10 selectively promotes dominance of polyclonally activated and environmental antigen-driven IFN-gamma over IL-4 responses, FASEB J 12 (1998), 705-713.

[15] J.H. Dufour, M. Dziejman, M.T. Liu, J.H. Leung, T.E. Lane and A.D. Luster, IFN-gamma-inducible protein 10 (IP-10; CXCL10)-deficient mice reveal a role for IP-10 in effector T cell generation and trafficking, J Immunol 168 (2002), 31953204.

[16] E. Ferrero, P. Biswas, K. Vettoretto, M. Ferrarini, M. Uguccioni, L. Piali et al., Macrophages exposed to Mycobacterium tuberculosis release chemokines able to recruit selected leucocyte subpopulations: focus on gammadelta cells, Immunology 108 (2003), 365-374.

[17] A. Azzurri, O.Y. Sow, A. Amedei, B. Bah, S. Diallo, G. Peri et al., IFN-gamma-inducible protein 10 and pentraxin 3 plasma levels are tools for monitoring inflammation and disease activity in Mycobacterium tuberculosis infection, Microbes Infect 7 (2005), 1-8.

[18] E. Whittaker, A. Gordon and B. Kampmann, Is IP-10 a better biomarker for active and latent tuberculosis in children than IFNgamma? PLoS One 3 (2008), e3901.

[19] M. Ruhwald, M. Bjerregaard-Andersen, P. Rabna, K. Kofoed, J. Eugen-Olsen and P. Ravn, CXCL10/IP-10 release is induced by incubation of whole blood from tuberculosis patients with 
ESAT-6, CFP10 and TB7.7, Microbes Infect 9 (2007), 806812.

[20] E. Lockhart, A.M. Green and J.L. Flynn, IL-17 production is dominated by gammadelta $\mathrm{T}$ cells rather than $\mathrm{CD} 4 \mathrm{~T}$ cells during Mycobacterium tuberculosis infection, J Immunol 177 (2006), 4662-4669.

[21] M.L. Michel, A.C. Keller, C. Paget, M. Fujio, F. Trottein, P.B. Savage et al., Identification of an IL-17-producing NK1.1(neg) iNKT cell population involved in airway neutrophilia, $J$ Exp Med 204 (2007), 995-1001.

[22] B. Stockinger, M. Veldhoen and B. Martin, Th17 T cells. linking innate and adaptive immunity, Semin Immunol 19 (2007), 353-361.

[23] T.J. Scriba, B. Kalsdorf, D.A. Abrahams, F. Isaacs, J. Hofmeister, G. Black et al., Distinct, specific IL-17- and IL-22producing CD4+ $\mathrm{T}$ cell subsets contribute to the human anti-mycobacterial immune response, J Immunol 180 (2008), 1962-1970.

[24] A. Poggi, S. Catellani, A. Musso and M.R. Zocchi, Gammadelta $\mathrm{T}$ lymphocytes producing ifngamma and IL-17 in response to Candida albicans or mycobacterial antigens: possible implications for acute and chronic inflammation, Curr Med Chem 16 (2009), 4743-4749.

[25] S. Tanaka, T. Yoshimoto, T. Naka, S. Nakae, Y. Iwakura, D. Cua et al., Natural occurring IL-17 producing T cells regulate the initial phase of neutrophil mediated airway responses, $J$ Immunol 183 (2009), 7523-7530.

[26] S.A. Khader and A.M. Cooper, IL-23 and IL-17 in tuberculosis, Cytokine 41 (2008), 79-83.

[27] M. Umemura, A. Yahagi, S. Hamada, M.D. Begum, H. Watanabe, K. Kawakami et al., IL-17-mediated regulation of innate and acquired immune response against pulmonary Mycobacterium bovis bacille Calmette-Guerin infection, J Immunol 178 (2007), 3786-3796.

[28] S.A. Khader, G.K. Bell, J.E. Pearl, J.J. Fountain, J. RangelMoreno, G.E. Cilley et al., IL-23 and IL-17 in the establishment of protective pulmonary $\mathrm{CD} 4+\mathrm{T}$ cell responses after vaccination and during Mycobacterium tuberculosis challenge, Nat Immunol 8 (2007), 369-377.
[29] H. Bai, J. Cheng, X. Gao, A.G. Joyee, Y. Fan, S. Wang et al., IL-17/Th17 promotes type $1 \mathrm{~T}$ cell immunity against pulmonary intracellular bacterial infection through modulating dendritic cell function, J Immunol 183 (2009), 5886-5895.

[30] K. Dheda, J.S. Chang, S. Lala, J.F. Huggett, A. Zumla and G.A. Rook, Gene expression of IL17 and IL23 in the lungs of patients with active tuberculosis, Thorax $\mathbf{6 3}$ (2008), 566-568.

[31] F.C. de Beer, A.E. Nel, R.P. Gie, P.R. Donald and A.F. Strachan, Serum amyloid A protein and C-reactive protein levels in pulmonary tuberculosis: relationship to amyloidosis, Thorax 39 (1984), 196-200.

[32] P. Maasilta and A.A. Kostiala, Serum levels of C-reactive protein in patients with pulmonary tuberculosis and malignant tumors of the chest, Infection 17 (1989), 13-14.

[33] G.M. Scott, P.G. Murphy and M.E. Gemidjioglu, Predicting deterioration of treated tuberculosis by corticosteroid reserve and C-reactive protein, J Infect 21 (1990), 61-69.

[34] F.G. Witebsky and P.S. Conville, The laboratory diagnosis of mycobacterial diseases, Infect Dis Clin North Am 7 (1993), 359-376.

[35] J.D. Christie and D.R. Callihan, The laboratory diagnosis of mycobacterial diseases: Challenges and common sense, Clin Lab Med 15 (1995), 279-306.

[36] H.P. McAdams, J. Erasmus and J.A. Winter, Radiologic manifestations of pulmonary tuberculosis, Radiol Clin North Am 33 (1995), 655-678.

[37] E.A. Nardell, Impact of DOTS and DOTS-plus on multidrug resistant TB: DOTS-plus strengthens, not weakens, DOTS programmes, BMJ 327 (2003), 164

[38] X. Chen, J. Wan, J. Liu, W. Xie, X. Diao, J. Xu et al., Increased IL-17-producing cells correlate with poor survival and lymphangiogenesis in NSCLC patients, Lung Cancer 2009; (Epub ahead of print).

[39] K. Nieminen, E. Valovirta and J. Savolainen, Clinical outcome and IL-17, IL-23, IL-27 and FOXP3 expression in peripheral blood mononuclear cells of pollen-allergic children during sublingual immunotherapy, Pediatr Allergy Immunol 2009; (Epub ahead of print). 


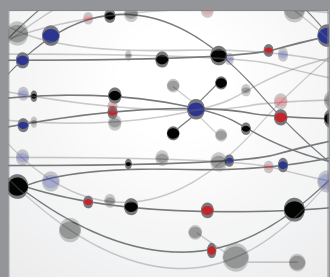

The Scientific World Journal
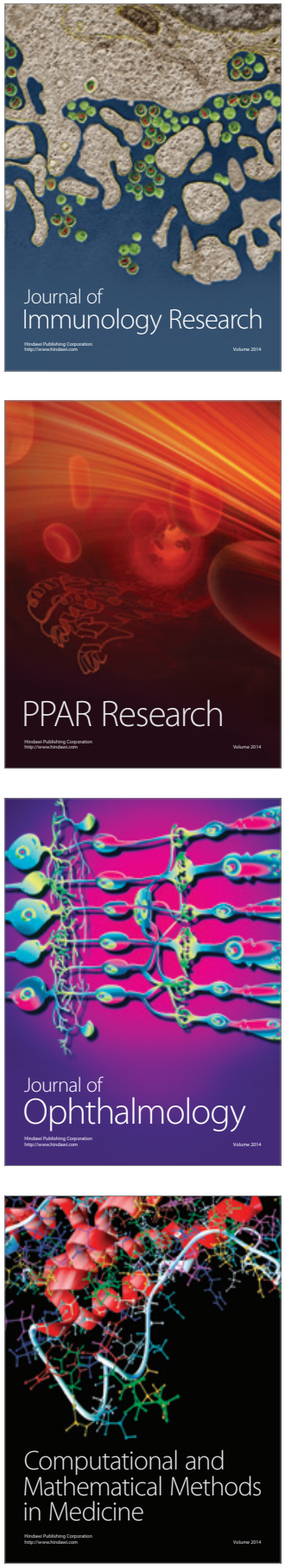

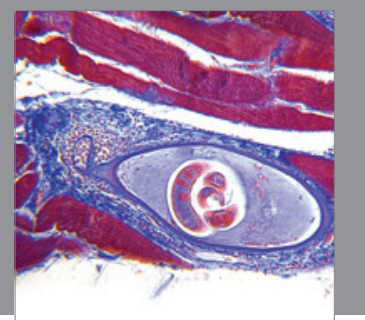

Gastroenterology

Research and Practice
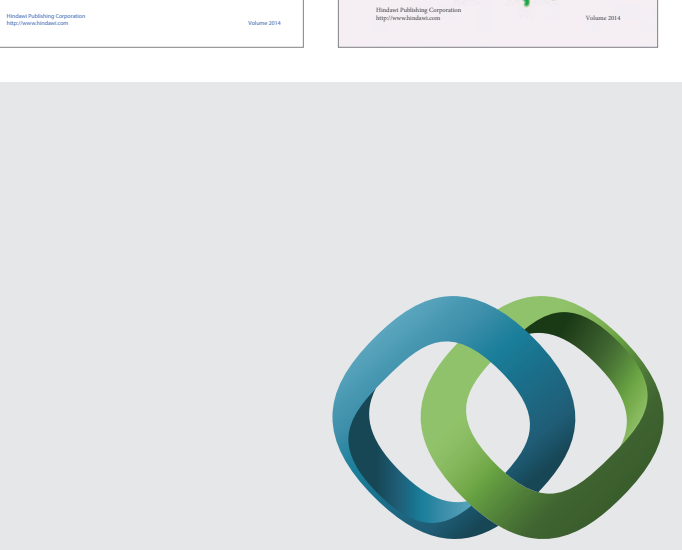

\section{Hindawi}

Submit your manuscripts at

http://www.hindawi.com
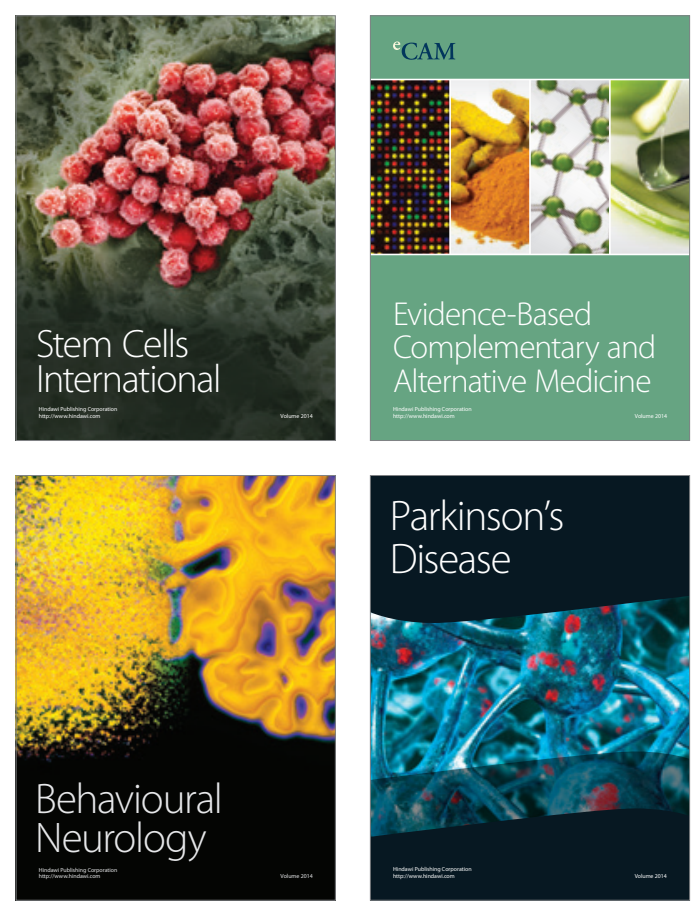

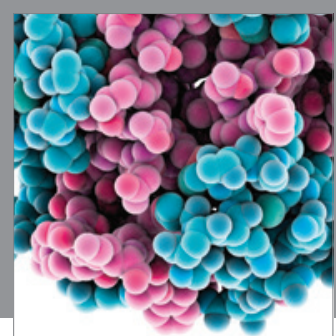

Journal of
Diabetes Research

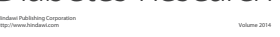

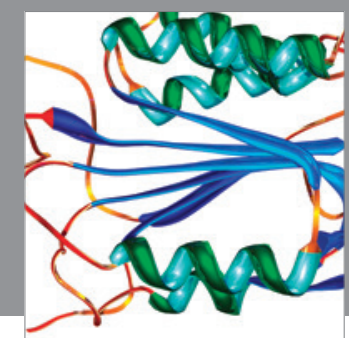

Disease Markers
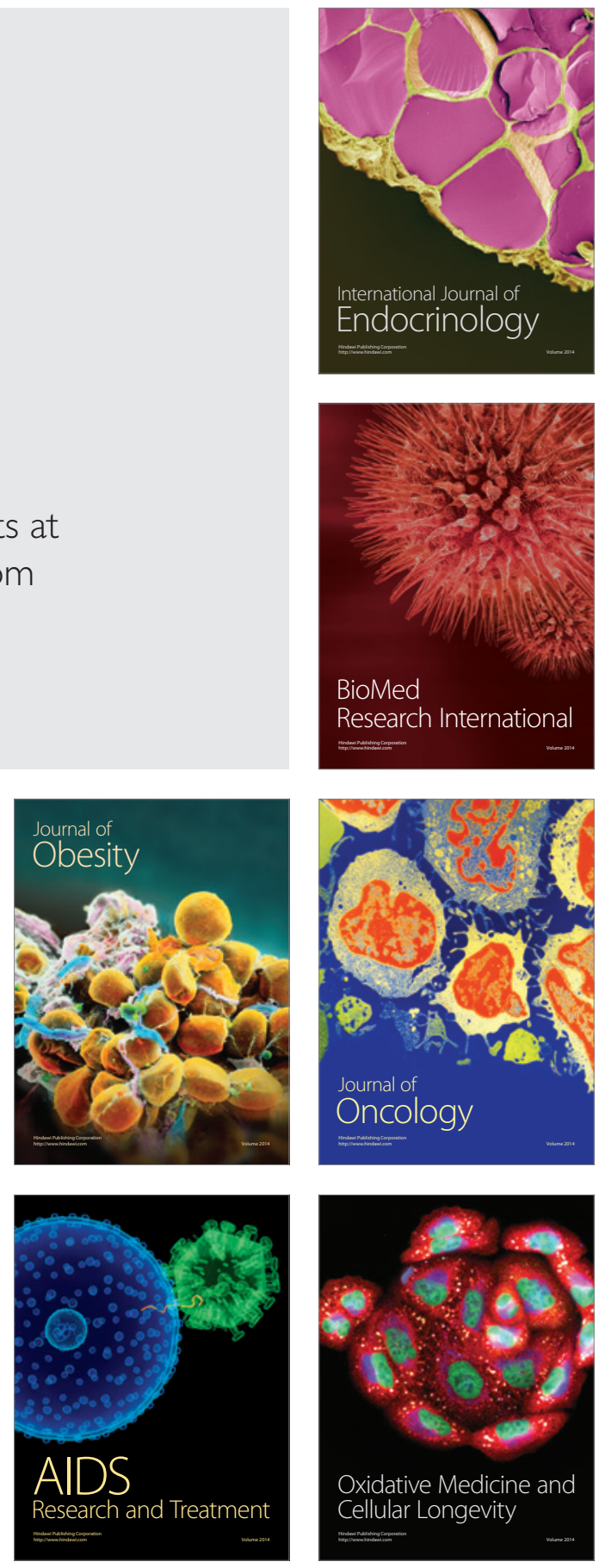